\begin{tabular}{c|c} 
CRWMS/M\&O & $\begin{array}{c}\text { Calculation Cover Sheet } \\
\text { Complete only applicable items. }\end{array}$
\end{tabular}

2. Calculation Title

Creating Input Tables From WAPDEG For RIP

3. Document Identifier (including Revision Number) B00000000-01717-0210-00013 REV 00

\begin{tabular}{|l|c|}
\hline $\begin{array}{l}\text { 5. Total Attachments } \\
0\end{array}$ & 6. Attachment Numbers - Number of pages \\
\hline 7. Originator & Kevin G. Mon \\
\hline 8. Checker & Bryan E. Bullard \\
\hline 9. Lead Design Engineer & Joon H. Lee \\
\hline 10. Remarks & \\
\hline
\end{tabular}

Uses TBV-95, 311, 323, 349, and 350.

Supporting electronic media $=$ MOL.19980716.0559.

\title{
Revision History
}

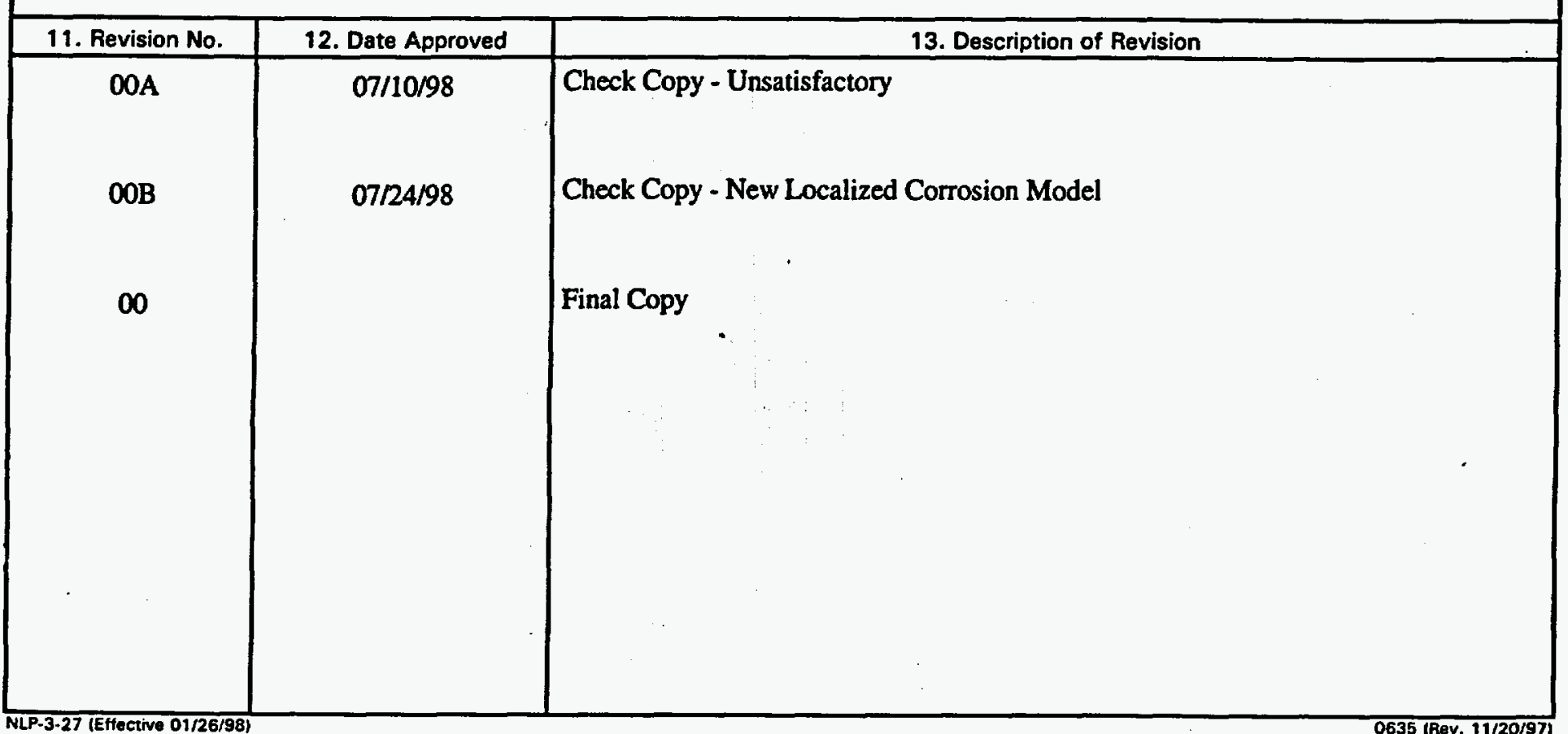


Title: $\quad$ Creating Input Tables From WAPDEG For RIP File: J:|QA|TSPA-VAOO|RIPTABLES-OO.DOC

Document Identifier: B00000000-01717-0210-00013 REV 00

Originator:

Kevin G. Mon

Checker:

Bryan E. Bullard

Lead Discipline Engineer: Joon H. Lee

Department Manager: Jerry A. McNeish

Draft Date:

August 10, 1998 
Title: Creating Input Tables From WAPDEG For RIP

\section{Table of Contents:}

Item

$\underline{\text { Page }}$

1.0 Purpose . .4

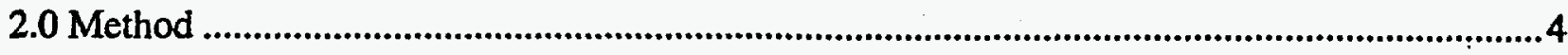

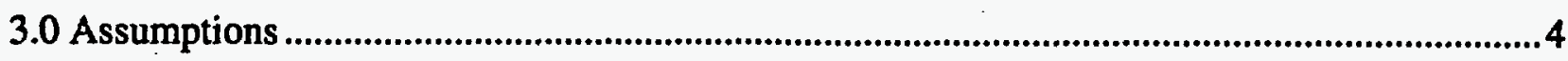

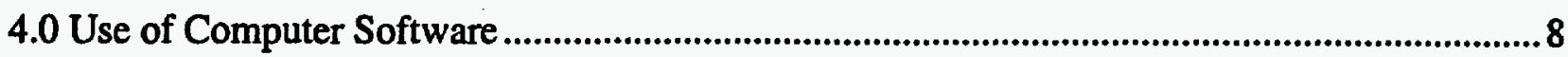

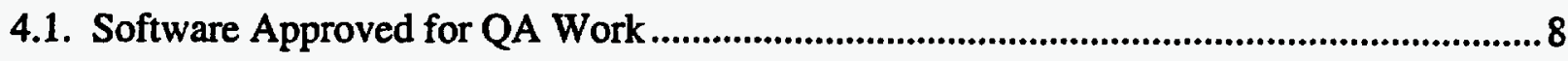

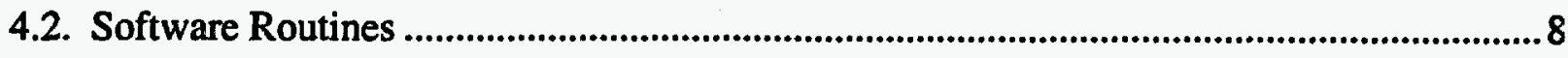

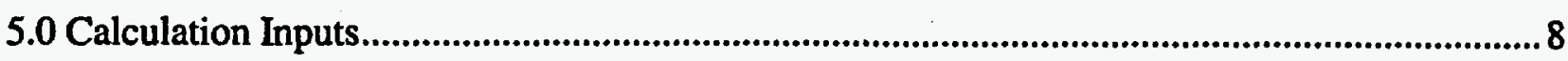

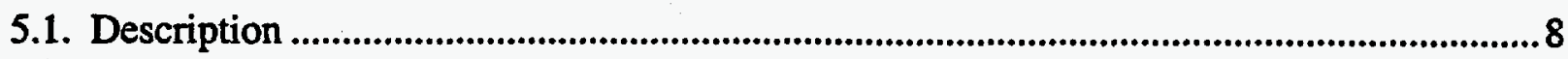

5.1.1. WAPDEG Input Files and Parameters Used .......................................................9

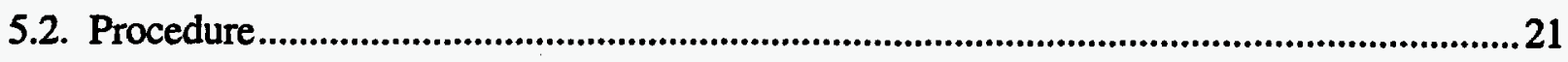

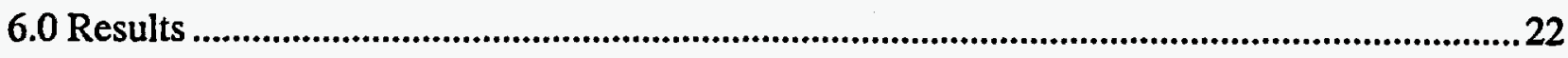

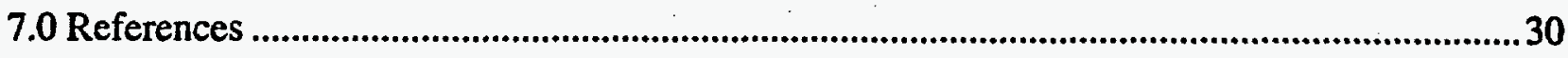

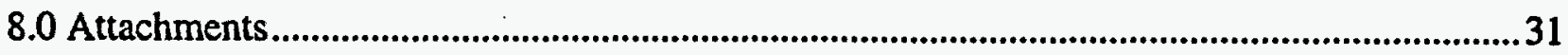




\subsection{Purpose}

The purpose of this calculation is to create tables for input into RIP ver. 5.18 (Integrated Probabilistic Simulator for Environmental Systems) from WAPDEG ver. 3.06 (Waste Package Degradation) output. This calculation details the creation of the RIP input tables for TSPA-VA REV. 00.

\subsection{Method}

Based on user-supplied input, the stochastic simulation code WAPDEG is used to generate waste package failure profiles. WAPDEG's inputs include time-varying histories of the temperature and relative humidity at the waste package surface, various temperature and relative humidity thresholds for corrosion initiation, corrosion models, and corrosion model parameter distributions. A waste package may fail either through localized corrosion processes (pitting or crevice corrosion), leading to small pin-hole perforations, or through general corrosion processes leading to much larger "patch" perforations. More detailed discussions of the WAPDEG conceptual model are given elsewhere (CRWMS M\&O, 1998a). The waste package failure profiles consist of time-varying measures of the number of pit and patch penetrations on each waste package. This information is abstracted by the WAPDEG post-processor, post306 (CRWMS M\&O, 1998b, Appendix D), to produce one RIP input table (Golder Associates, 1998) per WAPDEG simulation. The RIP input table contains:

1) The first failure (pit or patch) versus time curve for the waste packages to be simulated,

2) The average number of pits per failed waste package versus time curve and,

3) The average number of patches per failed waste package versus time curve.

Post306 has two main objectives:

a) It reformats the WAPDEG output to conform to the RIP input format and,

b) It decreases the number of points in each of the curves discussed above (1) through 3)) to approximately 82 (depends on the data being processed), through a process of time averaging.

More detailed discussions of post306 appear elsewhere (CRWMS M\&O, 1998b, Appendix D).

\subsection{Assumptions}

For the calculations involved in attaining a post processed table for input into RIP there are two steps to consider: 1) WAPDEG input and output and; 2) Post processing of WAPDEG output for creation of tables for input to RIP. There are several assumptions necessary to consider for the WAPDEG input and output. The following is a list of assumptions made in this process:

3.1 The variability in waste package degradation in a given repository region is adequately characterized by modeling 400 waste packages. This assumption is based on sensitivity studies performed with the WAPDEG code. This assumption is used in the WAPDEG input 
files (Section 5.1.1) in the fourth line after the last history file name.

3.2 The total surface area of the waste packages modeled is $298,862.5 \mathrm{~cm}^{2}$. This is based on the surface area of a 21 PWR (Pressurized Water Reactor) waste package type (Benton, 1997) (TBV-350). The length of this waste package is $5.335 \mathrm{~m}$ and the outer diameter is $1.664 \mathrm{~m}$. This total length includes 0.225 -m outer barrier extensions, for lifting of the waste package, on each end (i.e., two of them) which are not considered during corrosion modeling. Thus, the waste package surface area modeled (subject to corrosion) is given by:

$$
\left[\pi((5.335-2 \cdot 0.225) \cdot 1.664)+\frac{\pi}{2}(1.664)^{2}\right] \cdot\left(100^{2}\right)=298,862.5 \mathrm{~cm}^{2}
$$

This assumption is used only in determining the number of patches per waste package in Assumption 3.3.

3.3 Each waste package is composed of 964 patches each $310 \mathrm{~cm}^{2}$ in area. This patch size was chosen to be consistent with the size of a standard atmospheric corrosion test coupon (ASTM, 1992). This assumption is used in the WAPDEG input files (Section 5.1.1) in the fourth line after the last history file name.

3.4 There are 3,100 pits/patch on both the CAM and CRM yielding a pit density of 10 pits/ $\mathrm{cm}^{2}$. Pits are a modeling construct in WAPDEG where roughness factors and pit growth law parameters are applied. Hemispherical pits assumed in the CAM would overlap (at a 10 $\mathrm{pit} / \mathrm{cm}^{2}$ density) and produce the roughened general corrosion front described by the experts participating in the Waste Package Degradation Expert Elicitation (WPDEE - CRWMS M\&O, 1997). This assumption is used in the WAPDEG input files (Section 5.1.1) in the fourth line after the last history file name.

3.5 The top and bottom of the waste package are each defined with an angle of $180^{\circ}$. This is a conservative assumption as the CRM general corrosion rates under dripping are higher than the CRM general corrosion rates in the absence of dripping water (CRWMS M\&O, 1998c, 1998d). Dripping or pooling of dripped water can only occur on the top and bottom of the waste package and not along the sides (CRWMS M\&O, 1998b). Furthermore, localized corrosion of the CRM initiates only under dripping conditions. This assumption is used in the WAPDEG input files (Section 5.1.1) in the ninth and tenth lines after the last history file name.

3.6 If a waste package is dripped upon, $100 \%$ of its surface is contacted by the drips. Again this is a conservative assumption for the same reasons outlined in Assumption 3.5. This assumption is based on the assumption that seeps could move along the waste package length with time, thus potentially wetting the entire waste package surface. This assumption is used in the WAPDEG input files (Section 5.1.1) in the ninth and tenth lines after the last history file name. 
3.7 The RH thresholds for humid-air corrosion initiation and transition from humid-air to aqueous corrosion are perfectly rank correlated. The basis for this assumption is that the same factors (microstructural heterogeneity, the presence of salt films on the waste package surface, etc.) responsible for determining the humid-air corrosion initiation threshold would also factor into the determination of the RH threshold for the transition from humid-air to aqueous corrosion. Furthermore, as the CAM lasts only a few thousand years, this assumption has little effect on the overall waste package lifetime. This assumption is used in the [... Drip Features] input segments in the WAPDEG input files (Section 5.1.1). This input data is used on the seventh input line following the [No Drip Features] header and/or on the ninth input line after the [Neutral Drip Features] header.

3.8 The CAM pit multiple is represented by a bounded normal distribution with a mean of 1.5 and a standard deviation of 0.25 with a minimum value of 1.0. This assumption is based in part on expert elicitation (CRWMS M\&O, 1997) and in part on abstraction/analysis of literature observations (Marsh and Taylor, 1988; Marsh et. al., 1988). This assumption is used in the WAPDEG input files (Section 5.1.1) on the third input line after the [... Drip Model, CAM] header(s).

3.9 Galvanic protection is not operative. This assumption is based on the WPDEE (CRWMS M\&O, 1997). This assumption is used in the [. . . Drip Features] input segments in the WAPDEG input files (Section 5.1.1). This input data is used on the eighth input line following the [No Drip Features] header and/or on the tenth input line after the [Neutral Drip Features] header.

3.10 The CRM localized corrosion initiation is represented by a temperature threshold, which is distributed uniformly between 80 and $100^{\circ} \mathrm{C}$. This assumption is based on the expert elicitation of David Shoesmith (CRWMS M\&O, 1997) and is conservative relative to the elicitations of the other experts. This assumption is used in the [Neutral Drip Features] input segment in the WAPDEG input files (Section 5.1.1) on the third and fourth input lines following the [Neutral Drip Features] header.

3.11 The "ArtheniusPit" CRM localized corrosion model (CRWMS M\&O 1998g) (TBV-349) appropriately represents localized corrosion degradation of the CRM. The basis for this assumption is that this CRM localized corrosion model received almost universal support from the experts participating in the Waste Package Degradation Expert Elicitation (Pendleton, 1998, TBV-323). This assumption is used in the [Neutral Drip Model, CRM] input segment in the WAPDEG input files (Section 5.1.1) on the eleventh through sixteenth input lines following the [Neutral Drip Model, CRM] header.

3.12 Variability in waste package degradation is represented by dividing the total model variabilities equally among waste package-to-waste package variability and patch-to-patch variability. This assumption is used in the WAPDEG input files (Section 5.1.1) on the third line after the last history filename.

The bases for these assumptions are also extensively discussed elsewhere (CRWMS M\&O, 1998a). 
There are a few additional assumptions necessary to consider for the post-processing of WAPDEG output. The following is a list of assumptions made in this process:

3.13. If a non-dripping WAPDEG simulation is being post processed (defined by the fifth and sixth characters in the input filename prefix being "nd"), then the waste package diffusion start times (first penetration (pit or patch) in the bottom of the waste package) are considered to be the waste package failure times. The basis for this assumption is the assumption that radionuclide release is considered to occur only from the bottom part of the waste package. This assumption is used internally within Post 306.

3.14 If a dripping WAPDEG simulation is being post processed (defined by the fifth and sixth characters in the filename prefix being anything other than " $n$ d"), then the waste package advection start times are considered to be the waste package failure times. The advection time is the maximum of the earliest patch failure times amongst the top patches which are dripped upon and amongst bottom patches that are dripped upon or not. This time corresponds to the earliest time at which water can enter the waste package (from the top), interact with the waste form, and then leave the waste package (from the bottom) to enter the near field environment. The bases for this assumption are those mentioned above for Assumption 3.13; radionuclide release is considered to occur only from the bottom part of the waste package. The radionuclide release flux by advection is much greater than that due to diffusion, thus although basing waste package failure on advection time rather than diffusion time is slightly non-conservative, it is considered to yield a more accurate picture of radionuclide release characteristics. This assumption is used internally within Post306.

3.15 The waste package failure times are interpolated semi-logarithmically to the time grid used for WAPDEG's *.bin and *.pat files (where "*" represents the input file name prefix) (CRWMS M\&O. 1998b, Appendix D). This is done so that the average number of pits and patches per failed waste package can be calculated at each point of the *.pat and *.bin files' time grid. The interpolation is necessary as the waste package failure curve and the *.bin and *.pat files have different time grids. The basis of this assumption is that time values distributed over several orders of magnitude are well approximated with little loss of information. This assumption is used internally within Post306.

3.16 In the creation of input tables for RIP, it is desirable to decrease the number of data points supplied in the RIP input table. This is accomplished through a process of time averaging. First, the total number of waste packages that have failed during the total simulation time is determined. This value is divided by 80 to determine how many waste packages have failed at each of the 80 time bins (data points) which will be supplied to RIP. The average failure time, number of pits and patches per failed waste package is determined for each of the time bins. These are then written in a file conforming to the RIP table format (see RIP, 1998). Implicit in this process is the assumption that the original data (potentially greater than 80 data points) are well represented by the 80 data points supplied in the RIP input table. The basis for this assumption is the testing undertaken in Appendix D of the 
Title: Creating Input Tables From WAPDEG For RIP

WAPDEG Software Routine Report (CRWMS M\&O. 1998b). This assumption is used internally within Post306.

\title{
4.0 Use of Computer Software
}

\author{
4.1. Software Approved for QA Work \\ N/A.
}

\subsection{Software Routines}

The software used was WAPDEG ver. 3.06 and its post processor, post306 (CRWMS M\&O, 1998b). The following has been obtained from the Software Configuration Secretary (SCS) relative to this software:

Software Name:

Software Version:

CSCI Identifier:

Document Identifier:

Media Identifier:

Software Change Request:

\section{WAPDEG}

3.06

30048 v 3.06

30048-2999 Rev. 00

30048-M04-001 Rev. 00

LSBR 160

The WAPDEG simulations were executed on Hewlett-Packard HP-UX 20 workstations (CRWMSM\&O tags 102877, 112515, 108319, 107436, 107437, 108335, 111031). The post processing was accomplished on a Gateway 2000 equipped with a Pentium Pro $200 \mathrm{MHz}$ processor (CRWMS-M\&O tag 111033) in a Windows 95 operating system.

WAPDEG ver. 3.06 is an appropriate application because it is able to read input data and produce output files that can be post processed to create tables for input into RIP.

Post306 is an appropriate application because it is able to read input data and post process it to make tables for input into RIP.

\subsection{Calculation Inputs}

\subsection{Description}

Wap306 analyzes the inputs (*.inp, *..cdf, *.hst, see below) and creates several output files (*.aux, *.bin, *.cam, *.crm, *.out, *.pat). Post306 reads from the *.bin, *.pat, *.out files of the WAPDEG 
Title: Creating Input Tables From WAPDEG For RIP

ver. 3.06 runs and formulates the tables shown in Section 6.0 (CRWMS M\&O, 1998b).

The method of producing an output from WAPDEG for input into RIP entails the use of a number of files for the WAPDEG code to read. The following are the files required for WAPDEG:

1) Relative humidity (RH) and temperature histories at the surface of waste packages in a particular region of the repository (organized in bin numbers with file extension *.hst, where* is the filename prefix) (TBV-350).

2) Cumulative Distribution Functions (CDFs) for the temperature threshold (TThresh.cdf) (CRWMS M\&O, 1998c) for the onset of corrosion of the CAM (carbon steel outer barrier Corrosion Allowance Material).

3) CDFs for the RH threshold for the onset of humid air corrosion (HARH.cdf) and the transition from humid-air corrosion to aqueous corrosion (AQRH.cdf) (CRWMS M\&O, 1998d) for the corrosion allowance material (CAM) outer barrier.

4) Cumulative distribution functions (CDFs) for general corrosion rates under dripping for the inner barrier Corrosion Resistant Material (CRM) (CRWMS M\&O, 1998e) at 25,50 , and $100^{\circ} \mathrm{C}$ (gcrm98T1.cdf, gcrm98T2.cdf, and gcrm98T3.cdf, where $\mathrm{T} 1$ is $25^{\circ} \mathrm{C}, \mathrm{T} 2$ is $50^{\circ} \mathrm{C}$, and $\mathrm{T} 3$ is $100^{\circ} \mathrm{C}$ ).

5) CDFs for the CRM general corrosion rates with no drips (CRWMS M\&O, 1998f) at 25, 50, and $100^{\circ} \mathrm{C}$ (gcrmndT1.cdf, gcrmndT2.cdf, and gcrmndT3.cdf)

These file names and other model parameters are contained in the WAPDEG input file for the particular simulation being executed. The WAPDEG input file (*.inp) is read by wap306 (CRWMS M\&O, 1998b). The outputs resulting from WAPDEG simulations are then read by the post processor (post306 (CRWMS M\&O, 1998b, Appendix D)) which generates a table in a format appropriate for input into RIP (Golder Associates, 1998). The RIP input table contains:

1) The first failure (pit or patch) versus time curve for the waste packages to be simulated,

2) The average number of pits per failed waste package versus time curve and,

3) The average number of patches per failed waste package versus time curve.

\subsubsection{WAPDEG Input Files and Parameters Used}

Four WAPDEG input files were used to generate the TSPA-VA REV. 00 base case results; NEsfad100mh.inp, NEsfnd100mh.inp, SCsfad100mh.inp, and SCsfnd100mh.inp. The first two characters of the input file name indicate whether the North East (NE) or South Central (SC) region of the potential repository is being simulated. The next two characters (sf) indicate that the thermohydrologic histories appropriate for spent nuclear fuel are being used (TBV-350). If the next two characters are "nd," a no-drip case is being simulated; if they are "ad," the waste packages are subject to dripping throughout the simulation. The next three characters $(100)$ indicate what percentage of the waste package surface is contacted by drips (if they are present). The next characters $(\mathrm{mh})$ indicate that multiple histories for the relative humidity and temperature at the waste package surface are being used for the waste package groups in the simulation. 
1) NEsfad100mh.inp:

sf, always drip, 1008 dripped on, mult. hst, 6/26/98

No variability/uncertainty splitting

START OF PARAMETERS

3.06

12

NEsnf00noBFj2204.hst

15, 0 . 0 .

NEsnf01noBFj2204.hst

$59,0 ., 0$.

NEsnf10noBFj2204.hst

$6,0 \ldots 0$.

NEsnf11noBFj2204.hst

$120,0 \ldots 0$.

NEsnf12noBFj2204 .hst

$1,0 \ldots 0$.

NESnf21noBFj2204 hst

$60,0 \ldots 0$.

NEsnf22noBFj2204.hst

$16,0 \ldots 0$.

NEsnf31noBFj2204.hst

$26,0 ., 0$.

NEsnf32noBFj2204.hst

$50,0 \ldots 0$.

NEsnf42noBFj2204. hst

$36,0 \ldots 0$.

NEsnf52nOBFj2204. hst

$9,0.0$.

NEsnf62noBFj 2204 .hst

$2,0 \ldots 0$.

$10.0,2.0$

$75 \ldots, 0.5$

$400,964,3100,3100$

$1.0,1 . \mathrm{e} 6,1200$

$1 . e 4,5 . e 4,1 . e 5,1 . e 6$

431210

$0.0,0.0$

$180 ., 100$.

$180 ., 100$.

Fixed

0 .

Fixed

1000000 .

T, $\mathbf{F}$

Fixed

0.0

[No Drip Model, CAM]

CAMGeneral+PitMultiples

B-Normal

$1.5,0.25,1.0,1.0 \mathrm{e} 6$

[No Drip Model, CRM]

CRMGeneralRateonly

3 . $1 . e+6$

25.

File

gcrmndT1.cdf

50 .
|Version number of code

Number of alternate histories

History file

\# packages/group and Temp \& RH std deviations History file

\# packages/group and Temp \& RH std deviations

History file

\# packages/group and Temp \& RH std deviations

History file

\# packages/group and Temp \& RH std deviations

History file

\# packages/group and Temp \& RH std deviations

History file

\# packages/group and Temp \& RH std deviations

History file

\# packages/group and Temp \& RH std deviations History file

\# packages/group and Temp \& RH std deviations History file

\# packages/group and Temp \& RH std deviations History file

\# packages/group and Temp \& $\mathrm{RH}$ std deviations History file

\# packages/group and Temp \& RH std deviations History file

\# packages/group and Temp \& RH std deviations Thickness of outer, inner barriers $(\mathrm{cm})$

\& thick to fail CRM, frac variance to packs

Number of packs, patches/pack, pits/patch

Bin start time \& end time $(y)$, and \# of bins

Output times (y) for cumul. pit penetrations

Seed for random number generator

Max temp, $\mathrm{RH}$ change over a time step (C, 8RH)

Angle defining top(deg), \& seeing drips

Angle defining bottom(deg), \& seeing drips

Distribution for dripping start time

Distribution parameter (s)

Distribution for dripping stop time

Distribution parameter(s)

Neutral (T/F) water initially, new water (T/F)

Distr for time range for ceramic protection

Distribution parameter(s)

This segment always required

CAM corrosion model for no drips

Distribution for pit multiple

Mean, StDev, Min, Max

This segment always required

CRM corrosion model for drips

Number of dists (temps), max CRM rate

Temp appropriate for dist \#I

Distribution type for \#1

Distribution parameter (s)

Temp appropriate for dist \#1 
Title: Creating Input Tables From WAPDEG For RIP

File

gcrmndT2. cdf

100.

File

gcrmndT3.cdf

[No Drip Features]

File

TThresh.cdf

File

HARH. cdf

File

AQRH. cdf

1.0

$0.0,0.0$

0.0

$1.0,1.0$

[Neutral Drip Mode1, CAM] CAMGenera1+PitMultiples B-Normal

$1.5,0.25,1.0,1.0 \mathrm{e} 6$ [Neutral Drip Model, CRM]

CRMGenrate+ArrheniusPit

$3,1 . e+6$

25.

File

gcrm98T1.cdf

50.

File

germ98T2.cdf

100.

File

germ98T3.cdf

Normal

$11.275,2.4495$

Fixed

$5.5494 \mathrm{e}+003$

Fixed

0.5

[Neutral Drip Features]

File

TThresh.cdf

Uniform.

$80 . .100$.

File

HARH. $c \mathrm{df}$

File

AQRH.cdf

1.0

$0.0,0.0$

0.0

$1.0,1.0$
Distribution type for \#1

Distribution parameter (s)

Temp appropriate for dist \#2

Distribution type for \#2

Distribution parameter (s)

This segment always required

Distr for thermal protection temperature

Distribution parameter(s)

Dist type for humid-air initiation

Distribution parameter(s)

Dist type for humid-air/aqueous transition

Distribution parameter(s)

RH correlation factor

Galvanic protect depth 8 , \& patches protected

Spalling depth as a 8 of thickness

Multiples for CAM, CRM corrosion rates

Required if any non-neutral drips can be seen

CAM corrosion model for no drips

Distribution for pit multiple

Mean, StDev, Min, Max

Required if any non-neutral drips can be seen

CRM corrosion model for drips

Number of dists (temps), max CRM rate

Temp appropriate for dist \#1

Distribution type for \#1

Distribution parameter (s)

Temp appropriate for dist \#1

Distribution type for \#1

Distribution parameter ( $s$ )

Temp appropriate for dist \#2

Distribution type for \#2

Distribution parameter ( $s$ )

Distribution type for A (bo)

Distribution parameter(s)

Distribution type for $K$ (b1)

Distribution parameter(s)

Distribution type for $\mathrm{n}$

Distribution parameter (s)

Required if any non-neutral drips can be seen

Distr for thermal protection temperature

Distribution parameter(s)

Dist type for CRM LC T init

Distribution parameter

Dist type for humid-air initiation

Distribution parameter(s)

Dist type for humid-air/aqueous transition

Distribution parameter(s)

RH correlation factor

Galvanic protect depth $\&, \&$ patches protected

Spalling depth as a 8 of thickness

Multiples for CAM, CRM corrosion rates

\section{2) NEsfnd100mh.inp:}

mult hst, snf, $m=1.5$, no drip, $6 / 28 / 98$

No variability/uncertainty splitting 
Title: Creating Input Tables From WAPDEG For RIP

Page 12 of 31

3.06

12

NEsnf00nOBFj2204.hst

$15,0 ., 0$.

NESn f01nOBFj2204.hst

$59,0 ., 0$.

NEsnf10noBFj2204.hst

$6,0 \ldots, 0$.

NEsn f11nOBFj2204.hst

$120,0 \ldots 0$.

NEsnf12noBF $j 2204$.hst

$1,0 \ldots 0$.

NEsnf21nOBFj2204.hst

$60,0 ., 0$.

NEsnf22noBFj2204 .hst

$16,0 \ldots, 0$.

NEsnf31noBF $j 2204$.hst

$26,0 ., 0$.

NEsnf32noBFj2204 hst

$50,0 . .0$.

NEsnf 42 noBFj2204 hst

$36,0 ., 0$.

NEsnf52noBFj2204 .hst

$9,0.0$. 0.

NEsn f62noBFj2204 .hst

$2,0 ., 0$.

$10.0,2.0$

$75 . .0 .5$

$400,964,3100,3100$

$1.0,1 . e 6,1200$

$1 . e 4,1 . e 5,5 . e 5,1 . e 6$

431210

$0.0,0.0$

$180 . .0$.

$180 . .0$.

Fixed

0.0

[No Drip Model, CAM] CAMGeneral+PitMultiples B-Normal

$1.5,0.25,1.0,1.0 \mathrm{e} 6$

[No Drip Model, CRM]

CRMGeneralRateonly

$3,1 . e+6$

25.

File

gcrmndT1.cdf

50 .

File

gcrmndT2.cdf

100.

File

germat3.cdf

[No Drip Features]

File

TThresh.cdf

File

HARH. cdf

File

AQRH. cdf
IVersion number of code

|Number of alternate histories

/History file

|\# packages/group and Temp \& RH std deviations |History file

|\# packages/group and Temp \& RH std deviations |History file

|\# packages/group and Temp \& RH std deviations |History file

|\# packages/group and Temp \& RH std deviations |History file

|\# packages/group and Temp \& RH std deviations |History file

|\# packages/group and Temp \& RH std deviations |History file

|\# packages/group and Temp \& RH std deviations | History file

|\# packages/group and Temp \& RH std deviations |History file

|\# packages/group and Temp \& RH std deviations |History file

/ packages/group and Temp \& RH std deviations |History file

|\# packages/group and Temp \& RH std deviations | History file

|\# packages/group and Temp \& RH std deviations Thickness of outer, inner barriers $(\mathrm{cm})$

\& thick to fail CRM, frac variance to packs Number of packs, patches/pack, pits/patch Bin start time \& end time $(y)$, and \# of bins

Output times ( $y$ ) for cumul. pit penetrations I Seed for random number generator

(Max temp, RH change over a time step (C, 8RH)

Angle defining top(deg), \& seeing drips

Angle defining bottom(deg), \& seeing drips

Distr for time range for ceramic protection

Distribution parameter(s)

This segment always required

CAM corrosion model for no drips

Distribution for pit multiple

Mean, StDev, Min, Max

This segment always required

CRM corrosion model for drips

Number of dists (temps); $\max$ CRM rate

Temp appropriate for dist \#1

Distribution type for \#I

Distribution parameter (s)

Temp appropriate for dist \#1

Distribution type for \#1

Distribution parameter (s)

Temp appropriate for dist \#2

Distribution type for \#2

Distribution parameter ( $s$ )

This segment always required

Distr for thermal protection temperature

Distribution parameter(s)

Dist type for humid-air initiation

Distribution parameter(s)

Dist type for humid-air/aqueous transition

Distribution parameter(s) 
Title: Creating Input Tables From WAPDEG For RIP

1.0

$0.0,0.0$

0.0

$1.0,1.0$

[Neutral Drip Model, CAM]

CAMGeneral+PitMultiples

B-Normal

$1.5,0.25,1.0,1.0 \mathrm{e} 6$

[Neutral Drip Model, CRM]

CRMGenrate+ArrheniusPit

$3,1 . e+6$

25.

File

germ98T1.cdf

50.

File

germ98T2. cdf

100 .

File

gcrm98T3.cdf

Normal

$11.275,2.4495$

Fixed

$5.5494 e+003$

Fixed

0.5

[Neutral Drip Features]

File

TThresh.cdf

Uniform

$80 . .100$.

File

HARH.cdf

File

AQRH.cdf

1.0

$0.0,0.0$

0.0

$1.0,1.0$
RH correlation factor

Galvanic protect depth $\&$, \& patches protected

Spalling depth as a 8 of thickness

Multiples for CAM, CRM corrosion rates

Required if any non-neutral drips can be seen

CAM corrosion model for no drips

Distribution for pit multiple

Mean, StDev, Min, Max

Required if any non-neutral drips can be seen

CRM corrosion model for drips

Number of dists (temps), max CRM rate

Temp appropriate for dist \#1

Distribution type for \#1

Distribution parameter (s)

Temp appropriate for dist \#1

Distribution type for \#1

Distribution parameter (s)

Temp appropriate for dist \#2

Distribution type for \#2

Distribution parameter (s)

Distribution type for A (bo)

Distribution parameter(s)

Distribution type for $\mathrm{K}$ (b1)

Distribution parameter(s)

Distribution type for $\mathrm{n}$

Distribution parameter(s)

Required if any non-neutral drips can be seen

Distr for thermal protection temperature

Distribution parameter(s)

Dist type for CRM LC $T$ init

Distribution parameter

Dist type for humid-air initiation

Distribution parameter(s)

Dist type for humid-air/aqueous transition

Distribution parameter(s)

$\mathrm{RH}$ correlation factor

Galvanic protect depth $z, \&$ patches protected

Spalling depth as a $\&$ of thickness

Multiples for CAM, CRM corrosion rates

3) SCsfad100mh.inp:

snf, always drip, 1008, avg hst

$6 / 28 / 98$, No variability/uncertainty splitting

START OF PARAMETERS

3.06

9

SCsnf00nOBFj2204.hst

$28,0 ., 0$.

SCsnf01nOBFj2204.hst

$97,0 ., 0$.

sCsnf02noBFj2204.hst

$1,0 ., 0$.

SCsnf10noBFj2204.hst

$4,0 ., 0$.

SCsnf11noBFj2204.hst

$154,0 ., 0$.
Version number of code

|Number of alternate histories |History file

|\# packages/group and Temp \& RH std |History file

I\# packages/group and Temp \& RH std |History file

|\# packages/group and Temp \& RH std |History file

|\# packages/group and Temp \& RH std |History file

|\# packages/group and Temp \& RH std 
Title: Creating Input Tables From WAPDEG For RIP

SCsnf12noBFj2204. hst

23, $0 ., 0$.

SCsnf21noBFj2204 .hst

$25,0 \ldots 0$.

SCsnf22noBFj2204.hst

$61,0 \ldots 0$.

SCsnf32noBFj2204.hst

$7,0 ., 0$.

$10.0,2.0$

$75 \ldots 0.5$

$400,964,3100,3100$

1.0, 1.e6, 1200

1.e4, 5.e4, 1.e5, 1.e6

431210

$0.0,0.0$

$180 ., 100$

$180 ., 100$

Fixed

0 .

Fixed

1000000 .

T, F

Fixed

0.0

[No Drip Model, CAM]

CAMGeneral+PitMultiples

B-Normal

$1.5,0.25,1.0,1.0 \mathrm{e} 6$

[No Drip Mode], CRM]

CRMGeneralRateonly

$3,1, e+6$

25.

File

gcrmndT1. cdf

50 .

File

gcrmndt2. cdf

100 .

File

gcrmndT3 . cdf

[No Drip Features]

File

TThresh.cof

File

HARH . cdf

File

AQRH.cdf

1.0

$0.0,0.0$

0.0

1.0.1.0

[Neutral Drip Model, CAM] CAMGeneral+PitMultiples B-Norma1

$1.5,0.25,1.0,1.0 \mathrm{e} 6$

[Neutral Drip Model, CRM] CRMGenrate+ArrheniusPit

3. $1 . e+6$

25.

File
|History file

|\# packages/group and Temp \& RH std

|History file

|\# packages/group and Temp \& RH std

|History file

|\# packages/group and Temp \& $\mathrm{RH}$ std

History file

\# packages/group and Temp \& RH std

Thickness of outer, inner barriers $(\mathrm{cm})$

\& thick to fail CRM, frac variance to packs

Number of packs, patches/pack, pits/patch

Bin start time \& end time $(y)$, and \# of bins

Output times ( $y$ ) for cumul. pit penetrations

| Seed for random number generator

(Max temp, RH change over a time step (C, 8RH)

Angle defining top (deg), \& seeing drips

Angle defining bottom(deg), \& seeing drips

Distribution for dripping start time

Distribution parameter(s)

Distribution for aripping stop time

Distribution parameter(s)

Neutral (T/F) water initially, new water (T/F)

Distr for time range for ceramic protection

Distribution parameter(s)

This segment always required

CAM corrosion model for no drips

Distribution for pit multiple

Mean, StDev, Min, Max

This segment always required

CRM corrosion model for drips

Number of dists (temps), max CRM rate

Temp appropriate for dist \#1

Distribution type for \#1

Distribution parameter (s)

Temp appropriate for dist \#1

Distribution type for \#1

Distribution parameter (s)

Temp appropriate for dist \#2

Distribution type for \#2

Distribution parameter (s)

This segment always required

Distr for thermal protection temperature

Distribution parameter (s)

Dist type for humid-air initiation

Distribution parameter (s)

Dist type for humid-air/aqueous transition

Distribution parameter(s)

RH correlation factor

Galvanic protect depth $\&$, \& patches protected

Spalling depth as a \& of thickness

Multiples for CAM, CRM corrosion rates

Required if any non-neutral drips can be seen CAM corrosion model for no drips

Distribution for pit multiple

Mean, StDev, Min, Max

Required if any non-neutral drips can be seen

CRM corrosion model for drips

Number of dists (temps), max CRM rate

Temp appropriate for dist \#1

Distribution type for $\# 1$ 
Title: Creating Input Tables From WAPDEG For RIP

gcrm98T1.cdf
50.
File
gcrm98T2.cdf
100.
File
gcrm98T3.cdf
Normal
$11.275,2.4495$
Fixed
5.5494 e+003
Fixed
0.5
[Neutral Drip Features]
File
TThresh.cdf
Uniform
80. 100.
File
HARH.cdf
File
AQRH.cdf
1.0
$0.0,0.0$
0.0
$1.0,1.0$

\author{
Distribution parameter (s) \\ Temp appropriate for dist \#1 \\ Distribution type for \#1 \\ Distribution parameter (s) \\ Temp appropriate for dist \#2 \\ Distribution type for $\# 2$ \\ Distribution parameter (s) \\ Distribution type for A (b0) \\ Distribution parameter(s) \\ Distribution type for $\mathrm{K}$ (b1) \\ Distribution parameter(s) \\ Distribution type for $n$ \\ Distribution parameter(s) \\ Required if any non-neutral drips can be seen \\ Distr for thermal protection temperature \\ Distribution parameter(s) \\ Dist type for CRM LC T init \\ Distribution parameter \\ Dist type for humid-air initiation \\ Distribution parameter (s) \\ Dist type for humid-air/aqueous transition \\ Distribution parameter(s) \\ RH correlation factor \\ Galvanic protect depth \&, \& patches protected \\ Spalling depth as a of of thickness \\ Multiples for CAM, CRM corrosion rates
}

Average hst, snf, $m=1.5$, no drip

$6 / 28 / 98$, No variability/uncertainty splitting

START OF PARAMETERS

3.06

9

SCsnf00noBFj2204.hst

$28,0 \ldots 0$.

SCsnf01nOBFj2204.hst

97, 0 . 0 .

SCsnf02nOBFj2204.hst

$1,0 ., 0$.

SCsnf10nOBFj2204.hst

$4,0,0$.

SCsnfI1nOBFj2204.hst

$154,0 . .0$.

SCsnf12nOBFj2204.hst

$23,0 ., 0$.

SCsnf21nOBFj2204.hst

$25,0 \ldots, 0$.

SCsnf22noBFj2204.hst

$61,0 ., 0$.

SCsnf32noBFj2204.hst

$7,0 . .0$.

$10.0,2.0$

$75 \ldots, 0.5$

$400,964,3100,3100$

$1.0,1 . e 6,1200$

1.e4, 1.e5, 5.e5, 1.e6
Version number of code

|Number of alternate histories |History file

|\# packages/group and Temp \& RH std |History file

|\# packages/group and Temp \& RH std |History file

|\# packages/group and Temp \& RH std |History file

| \# packages/group and Temp \& RH std |History file

I\# packages/group and Temp \& RH std |History file

I\# packages/group and Temp \& RH std |History file

|\# packages/group and Temp \& RH std | History file

I\# packages/group and Temp \& RH std |History file

\# packages/group and Temp \& RH std

Thickness of outer, inner barriers (cm)

if thick to fail CRM, frac variance to packs

Number of packs, patches/pack, pits/patch

Bin start time \& end time $(y)$, and \# of bins

Output times (y) for cumul. pit penetrations 
Title: Creating Input Tables From WAPDEG For RIP

431210

$0.0,0.0$

$180 \ldots 0$.

$180 . .0$.

Fixed

0.0

[No Drip Model, CAM]

CAMGeneral+PitMultiples

B-Normal

$1.5,0.25,1.0,1.0 \mathrm{e} 6$

[No Drip Model, CRM]

CRMGeneralRateOnly

3 , $1 . e+6$

25.

File

germndT1.edf

50.

File

gcrmndT2 . cdf

100.

File

gcrmndT3.cdf

[No Drip Features]

File

TThresh.cdf

File

HARH . CdE

File

AQRH.cdf

1.0

$0.0,0.0$

0.0

$1.0,1.0$

[Neutral Drip Model, CAM]

CAMGeneral+PitMultiples

B-Normal

$1.5,0.25,1.0,1.0 \mathrm{e} 6$

[Neutral Drip Model, CRM]

CRMGenrate+Arrhenius $P$ it

$3,1 . e+6$

25.

File

germ98T1.cdf

50 .

File

gcrm98T2.cdf

100.

File

germ98T3.cdf

Normal

$11.275,2.4495$

Fixed

$5.5494 \mathrm{e}+003$

Fixed

0.5

[Neutra] Drip Features]

File

TThresh.cdf

Uniform

$80 ., 100$.
Seed for random number generator

Max temp, RH change over a time step (C, \&RH)

Angle defining top(deg), \& seeing drips

Angle defining bottom(deg), \& seeing drips

Distr for time range for ceramic protection

Distribution parameter(s)

This segment always required

CAM corrosion model for no drips

Distribution for pit multiple

Mean, StDev, Min, Max

This segment always required

CRM corrosion model for drips

Number of dists (temps), max CRM rate

Temp appropriate for dist \#1

Distribution type for \#1

Distribution parameter (s)

Temp appropriate for dist \#1

Distribution type for \#1

Distribution parameter (s)

Temp appropriate for dist \#2

Distribution type for $\# 2$

Distribution parameter (s)

This segment always reguired

Distr for thermal protection temperature

Distribution parameter(s)

Dist type for humid-air initiation

Distribution parameter(s)

Dist type for humid-air/aqueous transition

Distribution parameter(s)

RH correlation factor

Galvanic protect depth $8, \&$ patches protected

Spalling depth as a of thickness

Multiples for CAM, CRM corrosion rates

Required if any non-neutral drips can be seen

CAM corrosion model for no drips

Distribution for pit multiple

Mean, StDev, Min, Max

Required if any non-neutral drips can be seen

CRM corrosion model for drips

Number of dists (temps), $\max C R M$ rate

Temp appropriate for dist \#1

Distribution type for \#1

Distribution parameter (s)

Temp appropriate for dist \#1

Distribution type for \#1

Distribution parameter (s)

Temp appropriate for dist \#2

Distribution type for \#2

Distribution parameter (s)

Distribution type for A (bo)

Distribution parameter(s)

Distribution type for $\mathrm{K}$ (b1)

Distribution parameter(s)

Distribution type for $n$

Distribution parameter(s)

Required if any non-neutral drips can be seen

Distr for thermal protection temperature

Distribution parameter(s)

Dist type for CRM LC T init

Distribution parameter 
File

HARH . cdf

File

AQRH. cdf

1.0

$0.0,0.0$

0.0

$1.0,1.0$
Dist type for humid-air initiation

Distribution parameter(s)

Dist type for humid-air/aqueous transition

Distribution parameter(s)

RH correlation factor

Galvanic protect depth $\%$, \& patches protected

Spalling depth as a of thickness

Multiples for CAM, CRM corrosion rates

All of the text that appears above the 'START OF PARAMETERS' statement is copied into the output file (*.out, where the * represents the input file prefix) and can be used for any additional comments the user feels is necessary to distinguish the output. The relative humidity and temperature history information in the header of the input files for South Central (SC) regions is not correct. It should be "multiple histories."

The version number (3.06) is read and checked against the version of WAPDEG being executed; a mismatch in version numbers will halt program execution. The next line of the input file specifies the number of waste package groups (number of history files, 12 for the NE inputs and 9 for the SC inputs) which will be used to represent local repository conditions (spatial variability in the driftscale thermohydrology of the repository). The next 24 (NE) or 18 (SC) lines in the input file are composed of 12 (NE) or 9 (SC) pairs of input lines in which the first line specifies the name of the mean history file (*.hst, composed of three columns of data; time, waste package surface temperature, and relative humidity (TBV-350)) to be used for each waste package group followed by a line composed of three values; the number of waste packages that the history file is to be applied to (the number of waste packages in the group), and the standard deviations for allowed variations in T and RH from the waste package group's mean history file. In the TSPA-VA base case there are no variations allowed from the $\mathrm{T}$ and $\mathrm{RH}$ data given in the history file. The next input lines:

$10.0,2.0$

$75 ., 0.5$

$400,964,3100,3100$

$1.0,1, \mathrm{e} 6,1200$

$1 . e 4,1 . e 5,5 . e 5,1 . e 6$

431210

$0.0,0.0$
|Thickness of outer, inner barriers (cm) if thick to fail CRM, frac variance to packs Number of packs, patches/pack, pits/patch Bin start time \& end time $(y)$, and \# of bins Output times $(y)$ for cumul. pit penetrations Seed for random number generator

Max temp, $R H$ change over a time step (C, 8RH)

allow for input of the outer and inner barrier thicknesses in $\mathrm{cm}$. They are 10 and $2 \mathrm{~cm}$ thick, respectively (Benton, 1997) (TBV-350). The first value on the next line is related to determination of a 'structural failure time' for the waste package. For each waste package, WAPDEG calculates the average (over all patches) CRM general corrosion depth at each time step. When this average depth exceeds the given percentage (75\% in this case) of the total CRM thickness, the waste package can be considered to have structurally collapsèd (presumably due to static loads from its own weight, the weight of rock, etc.). This degradation mode is not included in the TSPA-VA Rev. 00 base case. WAPDEG still continues to model corrosion after this time and the structural failure time is output (to the *.out file) for possible use in other models. The second value on this line $(0.5)$ allows the user to specify the fraction of the total variability in the various model parameters should be assigned to waste package-to-waste package variability and 1 - (this value) is assigned to patch-to-patch variability.

The line following allows the user to specify the number of waste packages (400), patches/waste 
package (964), and pits/patch $(3,100)$ in both the CAM and CRM to be simulated. The next line contains three values related to the storing of temporal results (number of pit and patch penetrations with time). The start time corresponds to the beginning of the simulation; if the history file does not contain data for this starting time, the simulation is assumed to start with the earliest time step given in the history file. The same is not true for the end time as a time step is added to the end of the history with the same temperature and relative humidity as the last time step in the history file if the history file does not contain data for the simulation end time. The third value specifies how many (logarithmically distributed) time "bins" are used to store the cumulative pit penetration profiles. In this way, the resolution of the pit penetration profiles reported by WAPDEG is under user control.

The next line allows the user to specify up to 4 time values that are of particular interest. For each time value, WAPDEG will report (in the *.out file) the cumulative number of pit and patch penetrations in the time bin that contains the specified time value, and not the actual number of pit and patch penetrations at the exact time value specified. The following input line allows specification of the random number seed used to initialize the random number generator. The next line allows the user to enter the maximum allowed change in $T$ and $R H$ between time steps $(0.0,0.0$ causes this option to be ignored). WAPDEG will add time steps to the history file and interpolate $\mathrm{T}$ and $\mathrm{RH}$ data until these criteria are satisfied. The corrosion models used in WAPDEG require the application of constant environments for the duration of each time step. Through wise application of these values the user may increase the accuracy of the WAPDEG output. The next input lines:

180.100

$180 ., 100$.

Fixed

0 .

Fixed

1000000 .

T, F
Angle defining top(deg), \& seeing drips
Angle defining bottom(deg), o seeing drips
Distribution for dripping start time
Distribution parameter(s)
Distribution for dripping stop time
Distribution parameter(s)
Neutral(T/F) water initially, new water (T/F)

(T/F)

deal with application of dripping models; the user can enter the angles (degrees) that define the top and bottom of the waste package. The remaining angular range (if any) is defined to be the side of the waste package. The side of the waste package differs from the top or bottom in that it can never be dripped upon and thus never undergoes localized CRM corrosion. WAPDEG allows dripping on a given (top or bottom) patch to start sometime after the beginning of the simulation, to change corrosion model parameters (specified later in the input file as "Chemical" if it were used) sometime after that, and then for dripping to cease. The user must enter the percent of top and bottom patches that are dripped upon. In the TSPA-VA base case dripping simulations, the dripping surface percentage is fixed at a value of one hundred percent and the dripping water never changes chemistry. The word "Neutral" here has nothing to do with the actual water chemistry, it merely serves to identify a group of user-supplied input parameters that appear later in the input file. For the no-dripping WAPDEG simulations, the dripping surface percentage is set to zero and the dripping start, stop, and chemistry change (the last input line above) input lines must not be present in the input file, i.e.,

$180 \ldots$

$180 \ldots 0$.
Angle defining top(deg), \& seeing drips Angle defining bottom(deg), \& seeing drips 
Title: Creating Input Tables From WAPDEG For RIP

would be used in place of the seven input lines shown previously.

The next input lines:

Fixed

Distr for time range for ceramic protection

0.0

Distribution parameter(s)

are concerned with modeling the use of a ceramic coating to offer additional corrosion protection to the waste package. In the current WAPDEG version, the ceramic coating is considered to offer complete corrosion protection to the waste package until it fails. This parameter is not used in the TSPA-VA base case simulations.

The next input segment:

[No Drip Model, CAM] CAMGeneral+PitMultiples

B-Normal

$1.5,0.25,1.0,1.0 \mathrm{e} 6$

[No Drip Model, CRM]

CRMGeneralRateonly

$3,1 . e+6$

25.

File

gcrmndT1.cdf

50.

File

gcrmndT2 . cdf

100.

File

germndT3.cdf
This segment always required

CAM corrosion model for no drips

Distribution for pit multiple

Mean, StDev, Min, Max

This segment always required

CRM corrosion model for drips

Number of dists (temps), max CRM rate

Temp appropriate for dist \#1

Distribution type for \#1

Distribution parameter (s)

Temp appropriate for dist \#1

Distribution type for \#1

Distribution parameter (s)

Temp appropriate for dist \#2

Distribution type for \#2

Distribution parameter (s)

specifies the outer barrier (CAM) and inner barrier (CRM) corrosion models to be used when drips are not present. WAPDEG offers a variety of CAM and CRM models to choose from (CRWMS M\&O 1998b). Here we discuss only those used in the TSPA-VA analysis (CRWMS M\&O. 1998a).

The CAMGeneral+PitMultiples model for the CAM requires a roughness factor distribution. In the TSPA-VA base case, we make use of a bounded normal distribution with a mean of 1.5 , a standard deviation of 0.25 , a lower bound of one, and, effectively, no upper bound.

The CRMGeneralRateOnly model (as illustrated in the [No Drip, CRM] general corrosion input segment) requires specification of at least two (at most three) temperatures and general corrosion rate distributions (these can be any of the ones built in to WAPDEG). Here we use the no-drip Alloy 22 general corrosion rates derived in CRWMS M\&O, 1998d. WAPDEG 3.06 uses only the first two general corrosion rate distributions entered (CRWMS M\&O, 1998b). This oversight was fixed in WAPDEG 3.09 (CRWMS M\&O, 1998i). Note that the maximum CRM general corrosion rate is specified just after the number of distributions for the CRMGeneralRateOnly model.

The next set of input lines:

[No Drip Features]

File

TThresh.cdf

File

HARH . cdf
This segment always required

Distr for thermal protection temperature

Distribution parameter (s)

Dist type for humid-air initiation

Distribution parameter (s) 
File

AQRH. caf

1.0

$0.0,0.0$

0.0

Fixed

1.0

Fixed

1.0

1.0
Dist type for humid-air/aqueous transition

Distribution parameter (s)

RH correlation factor

Galvanic protect depth $\&$, \& patches protected

Spalling depth as a of thickness

Dist for multiple for CAM corrosion rate

Distribution parameter(s)

Dist for multiple for CRM corrosion rate

Distribution parameter(s)

Pack variance share for multiples

specify the 'No Drip Features.' These features include a distribution for the 'thermal protection temperature,' i.e., the temperature above which no corrosion is allowed to occur. Such a temperature threshold is used primarily because water will not exist in the liquid phase above this temperature, and thus no corrosion should occur. This threshold could be above the typical boiling point of water due to the presence of impurities in the water film on the waste package and variations in water chemistry. In this example, the temperature threshold is read from a file, TThresh.cdf (CRWMS M\&O, 1998e), resulting from values elicited from the Waste Package Degradation Expert Elicitation (CRWMS M\&O, 1997). WAPDEG also uses relative humidity (RH) thresholds (CRWMS M\&O, 1998f) for the onset of both humid-air and aqueous corrosion modes of the CAM.

The next input line allows the user to control the degree to which these RH thresholds are correlated. Entering a value of one forces the humid-air and aqueous RH thresholds into perfect rank correlation, i.e., if the aqueous RH threshold has a Cumulative Probability of 0.56 , the humid-air RH threshold with a Cumulative Probability of 0.56 will also be selected.

The 'galvanic protection depth \%' specifies the percent of the total CAM thickness that must remain in order for the CAM to afford galvanic protection to the '\% of patches protected' of the CRM. In this way the user can control the characteristics of the galvanic protection model used in WAPDEG. The subsequent input line allows specification of how thick a corrosion product film must be before it fails to adhere to the substrate and 'spalls' off the waste package surface. Neither galvanic protection nor spalling are used in the TSPA-VA base case. The 'Multiples for the CAM, CRM corrosion rates' are used in sensitivity studies to allow for modeling of corrosive environments that are considered to be more aggressive (multiples $>1$ ) or more benign (multiples $<1$ ) than those typically modeled in WAPDEG simulations. These are also not used (set to one) in the TSPA-VA base case.

The next three segments of the input file (and the three succeeding these, if there were a drip change time) are quite similar in form to the preceding three. The user enters [Neutral Drip Model, CAM], [Neutral Drip Model, CRM], and [Neutral Drip Features] in much the same manner as was done for the [No Drip . . . ] input segments. These may differ in salient parameters such as those used to specify the CRM general corrosion rates (CRWMS M\&O 1998c), RH thresholds, etc., but the form of the input is identical to that described above for the [No Drip ... .] sections with a few exceptions.

Immediately after specification of the three CDFs relevant to CRM general corrosion, the user must specify CRM localized corrosion parameters (CRWMS M\&O 1998g): 
Fixed

$5.5494 e+003$

Fixed

0.5
Distribution type for $k$ (b1)

Distribution parameter(s)

Distribution type for $n$

Distribution parameter(s)

Here, $\mathrm{A}$ has a mean of 11.275 , and a standard deviation of $2.4495, \mathrm{~K}$ is equal to 5549.4 , and $\mathrm{n}$ is 0.5 (Pasupathi, 1997, TBV-349).

The user must also supply two lines of input related to specification of a CRM temperature threshold for initiation of localized corrosion in the [Neutral Drip Features] section:

Uniform

$80 ., 100$.

Dist type for CRM LC $T$ init

Distribution parameter

These two lines appear immediately after specification of the temperature threshold for corrosion initiation. The CRM temperature threshold for initiation of localized corrosion is implemented such that if the temperature at the waste package surface is below the CRM temperature threshold for initiation of localized corrosion when the CAM is penetrated, no localized CRM corrosion occurs. However, once localized corrosion (pit or crevice corrosion) initiates, it continues regardless of the temperature, although at a temperature dependent rate.

To gain a deeper understanding of the WAPDEG code, the interested reader is directed to the Software Routine Report for WAPDEG (CRWMS M\&O 1998b).

\subsection{Procedure}

To run the WAPDEG simulations on the Hewlett-Packard HP-UX 20 workstations, it is necessary to create a directory containing all the necessary input files as well as the program itself (i.e., wap306). First it is necessary to compile the code in Fortran 77 in order to make it executable. This is done by typing, $\mathrm{f} 77+\mathrm{O} 2$ wap306.f -o <filenames on the UNIX command line. After compiling the program and importing all the above mentioned inputs, run the code by typing the name of the executable (in this case, wap306) on the UNIX command line and entering the name of the input file, i.e., NEsfad100mh.inp. If there are a large number of input files to be run, it is possible to run them sequentially using the UNIX script file, batchwpdg (CRWMS M\&O 1998b, Appendix C).

The 'raw' output from WAPDEG consists of five files: a *.out file, a *.pat file, a *.bin file, a *.crm file, and a *aux file (where* ${ }^{*}$ the input file (*.inp) name prefix). These files are included in the electronic media supporting this calculation (CRWMS M\&O, 1998h) and their content and format are discussed in the WAPDEG Software Routine Report (CRWMS M\&O, 1998b, Section 4.1). Only the *.out (waste package failure curves), *.pat (cumulative number of patch penetrations for each waste package), and *.bin (cumulative number of pit penetrations for each waste package) files are used to create the RIP input tables.

In order to create the tables for input to RIP, post306 is executed in a Windows DOS window within the same directory as the output files from WAPDEG (i.e., *.bin, *.pat, *.out). The program prompts the user for the particular filename prefix that is common to the WAPDEG simulation output files 
to be post processed. After the program post processes the WAPDEG output, it prompts the user to enter a table number thus creating the tables shown in Section 6.0. The output from the post processor consists of three files; *.asc, *.dat, and table.txx, where xx represents the user-input table number. These files are included in the electronic media supporting this calculation (CRWMS M\&O 1998h) and their content and format are discussed in the WAPDEG Software Routine Report (CRWMS M\&O, 1998b, Appendix D).

\subsection{Results}

As this calculation uses input data and assumptions that are "to be verified" (TBV-350) as well as the results of several other calculations that use input data and assumptions that are "to be verified" (CRWMS M\&O 1998c through 1998g, TBV 95, 311, 323, and 349) the results of this calculation should be considered "to be verified." The RIP input tables presented below are also included in the electronic media supporting this calculation (CRWMS M\&O 1998h) and their content and format are discussed in the RIP - Theory Manual and User's Guide (Golder Associates, 1998).

Below are shown the final RIP input tables for TSPA-VA base case REV. 00 created from WAPDEG version 3.06 and post processed by Post 306 .

NE-SNF-Dripping Case - table.t01

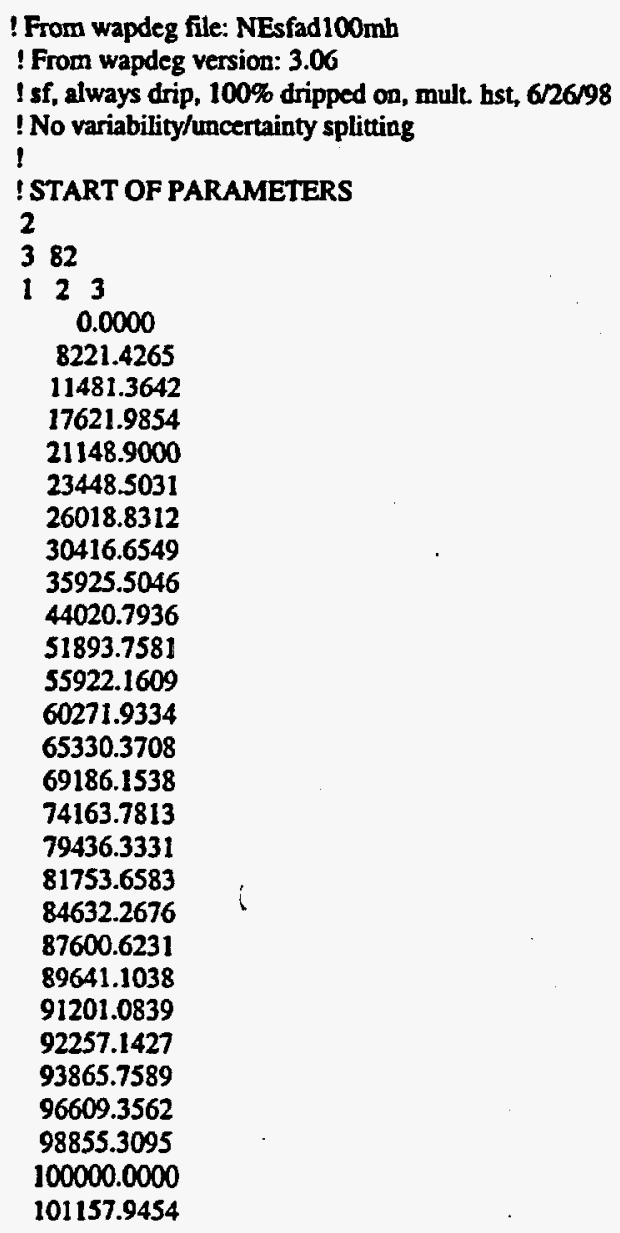


Title: Creating Input Tables From WAPDEG For RIP

Document Identifier: B00000000-01717-0210-00013 REV 00 (August 10, 1998)

Page 23 of 31

105953.4550

112206.8028

115480.1118

117489.7555

118850.2227

120226.4435

123747.3699

127350.3081

129570.8165

133358.0351

138044.5254

143726.2112

148767.2022

154005.4100

159406.9292

164066.2259

168852.3835

171790.8387

175800.1284

180928.5887

184077.2001

186208.7137

188364.9089

190546.0718

192752.4913

196113.3668

199526.2315

203005.2154

207733.8143

211348.9040

213796.2090

216271.8524

220042.8167

225168.2723

231749.7039

242693.1748

251188.6432

258544.9011

269165.3724

281875.6515

298577.8339

318114.8515

334980.2389

342767.7865

356930.7339

382458.1720

398107.1706

421882.8437

476258.7133

527939.2425

579078.4824

732241.1528

896569.4675

1000000.0000

0.0000

0.0000

0.0065

0.0176

0.0290

0.0456

0.0549

0.0686

0.0815

0.0915

0.1032

0.1169

0.1320

0.0000

0.0000

0.3406

0.6783

0.4396

0.2748

0.2291

0.1824

0.1537

0.1367

0.1213

0.1070

0.0000

0.0000

2.1008

5.9304

6.3432

5.1257

5.5135

5.9157

6.6558

7.6595

8.5835

0.1412

0.0947

8.5851

9.0072

10.6269 
Title: Creating Input Tables From WAPDEG For RIP

\begin{tabular}{|c|c|c|}
\hline 0.1564 & 0.0960 & 10.9901 \\
\hline 0.1654 & 0.1159 & 11.9210 \\
\hline 0.1821 & 0.1099 & 12.7122 \\
\hline 0.1955 & 0.1023 & 12.8606 \\
\hline 0.2055 & 0.1064 & 13.6106 \\
\hline 0.2188 & 0.1142 & 14.1921 \\
\hline 0.2308 & 0.1083 & 14.7705 \\
\hline 0.2495 & 0.1102 & 14.5912 \\
\hline 0.2609 & 0.1150 & 14.4418 \\
\hline 0.2675 & 0.1401 & 14.9307 \\
\hline 0.2822 & 0.1447 & 15.5018 \\
\hline 0.3084 & 0.1540 & 15.1503 \\
\hline 0.3135 & 0.1515 & 15.4715 \\
\hline 0.3203 & 0.1639 & 15.5874 \\
\hline 0.3268 & 0.2670 & 17.0103 \\
\hline 0.3405 & 0.1346 & 18.7265 \\
\hline 0.3591 & 0.1463 & 18.9402 \\
\hline 0.3767 & 0.1394 & 18.6999 \\
\hline 0.3839 & 0.1368 & 18.7153 \\
\hline 0.3927 & 0.1337 & 18.7665 \\
\hline 0.4040 & 0.1623 & 19.3713 \\
\hline 0.4234 & 0.1712 & 19.8454 \\
\hline 0.4294 & 0.1921 & 20.2638 \\
\hline 0.4384 & 0.2791 & 21.0156 \\
\hline 0.4500 & 0.4191 & 22.0391 \\
\hline 0.4635 & 0.3470 & 23.0576 \\
\hline 0.4790 & 0.2451 & 23.8855 \\
\hline 0.4902 & 0.4018 & 24.9547 \\
\hline 0.5035 & 0.6176 & 25.8439 \\
\hline 0.5132 & 0.3214 & 26.8708 \\
\hline 0.5266 & 0.4342 & 28.1724 \\
\hline 0.5415 & 0.5863 & 28.5532 \\
\hline 0.5508 & 1.0198 & 29.6750 \\
\hline 0.5653 & 0.6506 & 31.5159 \\
\hline 0.5835 & 0.4713 & 32.3637 \\
\hline 0.6008 & 0.5992 & 32.6542 \\
\hline 0.6110 & 0.9943 & 33.3968 \\
\hline 0.6206 & 0.6123 & 34.1980 \\
\hline 0.6275 & 1.0677 & 35.0058 \\
\hline 0.6395 & 1.4433 & 36.2309 \\
\hline 0.6581 & 1.8235 & 36.9139 \\
\hline 0.6637 & 1.1174 & 38.3982 \\
\hline 0.6732 & 0.4952 & 40.4611 \\
\hline 0.6931 & 0.3246 & 41.0051 \\
\hline 0.7017 & 0.3385 & 41.5818 \\
\hline 0.7112 & 0.3902 & 42.0424 \\
\hline 0.7214 & 0.5299 & 43.0337 \\
\hline 0.7346 & 1.0742 & 44.2079 \\
\hline 0.7447 . & 0.8248 & 46.0063 \\
\hline 0.7553 & 0.9370 & 48.8585 \\
\hline 0.7726 & 0.6569 & 50.3998 \\
\hline 0.7829 & 0.4636 & 51.7200 \\
\hline 0.7933 & 0.9268 & 53.9907 \\
\hline 0.8052 & 0.5520 & 56.3549 \\
\hline 0.8166 & 0.7697 & 59.3021 \\
\hline 0.8288 & 0.6568 & 62.4633 \\
\hline 0.8426 & 0.7629 & 64.7057 \\
\hline 0.8580 & 1.4569 & 64.9119 \\
\hline 0.8661 & 0.7490 & 66.8419 \\
\hline 0.8789 & 1.6763 & 69.9986 \\
\hline 0.8958 & 1.2252 & 71.0568 \\
\hline 0.9022 & 1.6194 & 74.1417 \\
\hline 0.9140 & 1.5212 & 81.0134 \\
\hline 0.9270 & 0.6100 & 86.8211 \\
\hline 0.9404 & 0.6645 & 91.4935 \\
\hline 0.9480 & 0.5847 & 100.5434 \\
\hline 0.9635 & 0.2530 & 102.8425 \\
\hline 0.9825 & 0.2545 & 102.3664 \\
\hline
\end{tabular}


NE-SNF-No Dripping Case - table.t02

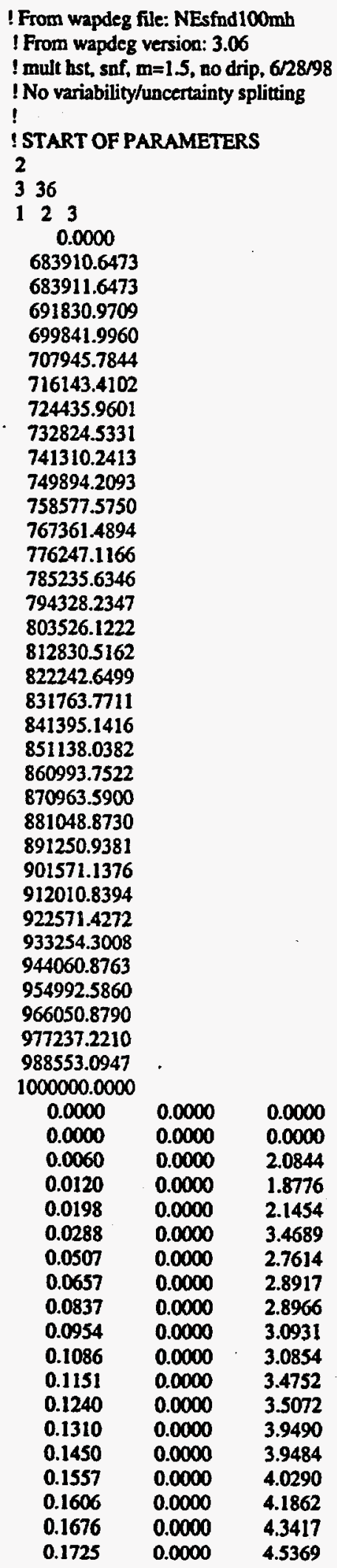




\section{Performance Assessment Operations}

Title: Creating Input Tables From WAPDEG For RIP

Document Identifier: B00000000-01717-0210-00013 REV 00 (August 10, 1998)

Page 26 of 31

$\begin{array}{lll}0.1819 & 0.0000 & 4.6462 \\ 0.1885 & 0.0000 & 4.7620 \\ 0.1953 & 0.0000 & 4.9282 \\ 0.2002 & 0.0000 & 5.1578 \\ 0.2047 & 0.0000 & 5.3602 \\ 0.2151 & 0.0000 & 5.3002 \\ 0.2181 & 0.0000 & 5.4909 \\ 0.2201 & 0.0000 & 5.7145 \\ 0.2223 & 0.0000 & 5.8693 \\ 0.2269 & 0.0000 & 6.1040 \\ 0.2329 & 0.0000 & 6.1829 \\ 0.2421 & 0.0000 & 6.2263 \\ 0.2440 & 0.0000 & 6.3844 \\ 0.2465 & 0.0000 & 6.5730 \\ 0.2491 & 0.0000 & 6.7247 \\ 0.2550 & 0.0000 & 6.7648 \\ 0.3150 & 0.0000 & 5.4762\end{array}$

SC-SNF-Dripping Case - table.t03

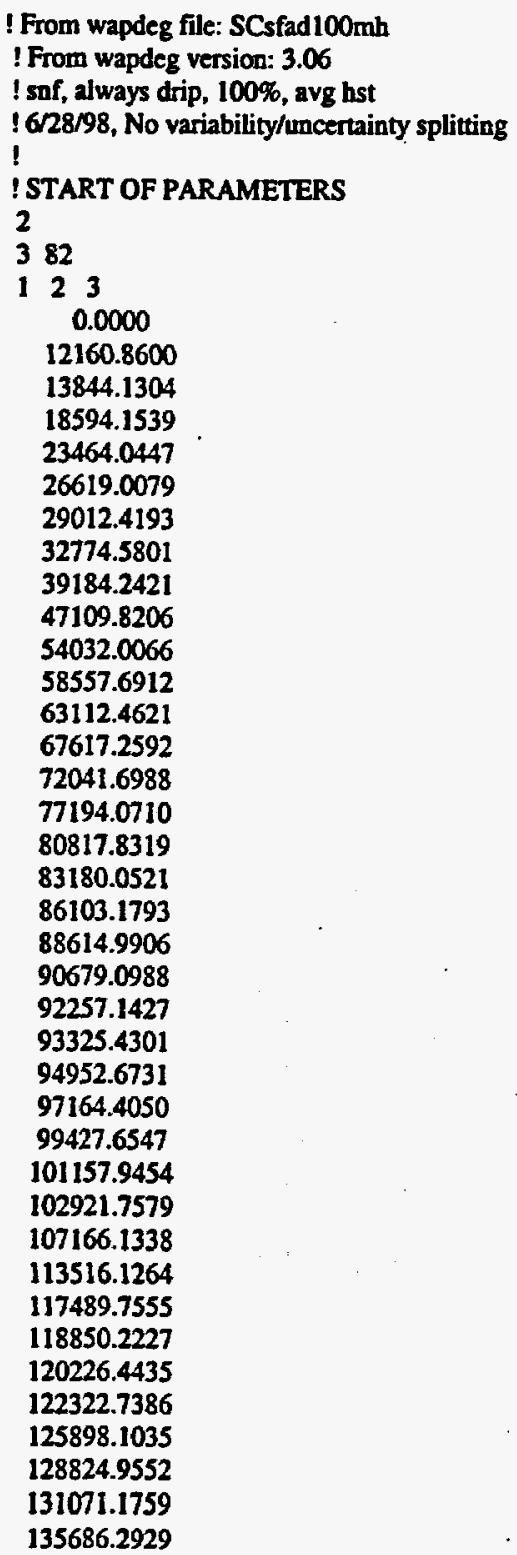


Title: Creating Input Tables From WAPDEG For RIP

\begin{tabular}{|c|c|c|}
\hline 0.2776 & 0.0585 & 15.4259 \\
\hline 0.2984 & 0.0628 & 15.3394 \\
\hline 0.3103 & 0.0644 & .4912 \\
\hline 0.3176 & 0.0826 & 15.8199 \\
\hline 0.3274 & 0.1678 & 16.8782 \\
\hline 0.3416 & 0.0366 & 18.5598 \\
\hline 0.3726 & 0.0335 & 18.2703 \\
\hline 0.3745 & 0.0467 & 18.5894 \\
\hline 0.3827 & 0.0457 & 18.6313 \\
\hline 0.3918 & 0.0510 & 18.830 \\
\hline 0.4055 & 0.06 & \\
\hline 0.4237 & 0.0649 & .597 \\
\hline 0.4284 & 0.0729 & 20.154 \\
\hline 0.4372 & 0.0957 & 21.202 \\
\hline 0.4518 & 0.1162 & 22.038 \\
\hline 0.4633 & 0.1403 & 22.593 \\
\hline 0.4763 & 0.2303 & 23.773 \\
\hline 0.4900 & 0.6017 & 24.968 \\
\hline 0.5034 & 0.6307 & 25.395 \\
\hline 0.5131 & 0.2961 & 26.434 \\
\hline 0.5266 & 0.5097 & 28.1268 \\
\hline 0.5389 & 0.2783 & 28.7436 \\
\hline 0.5481 & 1.0901 & 29.929 \\
\hline 0.5693 & 2.1024 & 31.553 \\
\hline 0.5870 & 0.1278 & 32.522 \\
\hline 0.6055 & 0.1486 & 32.866 \\
\hline 0.6098 & 0.1763 & 33.8370 \\
\hline 0.6223 & 0.2491 & 34.3952 \\
\hline 0.6291 & 0.4888 & 35.2831 \\
\hline 0.6411 & 0.1638 & 35.8612 \\
\hline 0.6515 & 0.1886 & 36.9900 \\
\hline 0.6624 & 0.1339 & 38.9037 \\
\hline 0.6781 & 0.2959 & 40.3916 \\
\hline 0.6897 & 0.1160 & 41.4037 \\
\hline 0.7080 & 0.1271 & 41.3445 \\
\hline 0.7120 & 0.15 & 42.1 \\
\hline 0.7232 & 0.1832 & 43.0416 \\
\hline 0.7336 & 0.3349 & 44.7681 \\
\hline 0.7454 & 0.5885 & 46.8160 \\
\hline 0.7564 & 0.2322 & 48.7363 \\
\hline 0.7717 & 0.8675 & 49.8935 \\
\hline 0.7829 & 0.4756 & 51.6145 \\
\hline 0.7909 & 0.2192 & 54.0333 \\
\hline 0.8041 & 0.50 & 56.6072 \\
\hline 0.8156 & 0.6728 & 59.5227 \\
\hline 0.8270 & 0.5638 & 61.9606 \\
\hline 0.8401 & 0.5995 & 64.2780 \\
\hline 0.8566 & 0.7964 & 65.1042 \\
\hline 0.8672 & 0.3403 & 66.7914 \\
\hline 0.8779 & 0.6725 & 69.7417 \\
\hline 0.8940 & 0.4614 & 70.8779 \\
\hline 0.9021 & 0.6406 & 73.7782 \\
\hline 0.9136 & 0.3784 & 80.6011 \\
\hline 0.9257 & 0.2095 & 86.3440 \\
\hline 0.9392 & 0.4621 & 90.9035 \\
\hline 0.9477 & 0.2058 & 100.293 \\
\hline 0.9635 & 0.0473 & 102.4898 \\
\hline .9825 & 0.0967 & 101.442 \\
\hline
\end{tabular}

SC-SNF-No Dripping Case - table.t04

! From wapdeg file: SCsfind $100 \mathrm{mh}$

! From wapdeg version: 3.06

! Average hst, snf, $m=1.5$, no drip

! 62898, No variability/uncerainty splitting

!

! START OF PARAMETERS 
Title: Creating Input Tables From WAPDEG For RIP

329

123 0.0000

741309.2413

741310.2413

749894.2093

758577.5750

767361.4894

776247.1166

785235.6346

794328.2347

803526.1222

812830.5162

822242.6499

831763.7711

841395.1416

851138.0382

860993.7522

870963.5900

881048.8730

891250.9381

901571.1376

912010.8394

922571.4272

933254.3008

944060.8763

954992.5860

966050.8790

977237.2210

988553.0947

1000000.0000

0.0000

0.0000

0.0104

0.0214

0.0365

0.0450

0.0597

0.0761

0.0936

0.1057

0.1116

0.1222

0.1330

0.1438

0.1515

0.1546

0.1638

0.1682

0.1696

0.1808

0.1867

0.1953

0.2045

0.2081

0.2092

0.2137

0.2194

0.2274

0.2900
$0.0000 \quad 0.0000$

$0.0000 \quad 0.0000$

$0.0000 \quad 3.8537$

$0.0000 \quad 3.2637$

$0.0000 \quad 2.3982$

$0.0000 \quad 2.4461$

$0.0000 \quad 2.8036$

$0.0000 \quad 2.6924$

$0.0000 \quad 2.7504$

$0.0000 \quad 2.7917$

$0.0000 \quad 3.0465$

$0.0000 \quad 3.2312$

$0.0000 \quad 3.3831$

$0.0000 \quad 3.5300$

$0.0000 \quad 3.5805$

$0.0000 \quad 4.0111$

$0.0000 \quad 4.1204$

$0.0000 \quad 4.2500$

$0.0000 \quad 4.4948$

$0.0000 \quad 4.4930$

$0.0000 \quad 4.6862$

$0.0000 \quad 4.8377$

$0.0000 \quad 4.9278$

$0.0000 \quad 5.0699$

$0.0000 \quad 5.2583$

$0.0000 \quad 5.3699$

$0.0000 \quad 5.4250$

$0.0000 \quad 5.5188$

$0.0000 \quad 4.3276$ 
Title: Creating Input Tables From WAPDEG For RIP

Document Identifier: B00000000-01717-0210-00013 REV 00 (Auguss 10, 1998)

Page 30 of 31

\subsection{References}

ASTM 1992. "Standard Practice for Conducting Atmospheric Corrosion Tests on Metals." G50-76. 1992 Annual Book of ASTM Standards: Metals Test Methods and Analytical Procedures: Wear and Erosion; Metal Corrosion, 3.02, § 3, 194-198. Philadelphia, Pennsylvania: ASTM.

Benton, H.A. 1997. Waste Package Data Input to TSPA-VA. CRWMS M\&O Interoffice Correspondence, LV.WP.JAC.04/97.081, April 14. TBV-350.

CRWMS M\&O 1998a. Preliminary Draft Chapter on Waste Package Degradation Abstraction and Testing for TSPA-VA. B00000000-01717-2200-00196 Rev. 00. Las Vegas, Nevada: Lee, J.H, Mon, K.G., Longsine, D.E, and Bullard, B.E.

CRWMS M\&O 1998b. Software Routine Report for WAPDEG (Version 3.06). DI: 30048-2999 REV 00. Las Vegas, Nevada: Mon, K.G.

CRWMS M\&O 1998c. Cumulative Distribution Functions for the Dripping Case of the Corrosion Resistant Material General Corrosion Model. B00000000-01717-0210-00014 Rev. 00. Las Vegas, Nevada: Mon, K.G. TBV-95.

CRWMS M\&O 1998d. Cumulative Distribution Functions for No Drip Corrosion Resistant Material General Corrosion Model. B00000000-01717-0210-00012 Rev. 00. Las Vegas, Nevada: Mon, K.G. TBV-95.

CRWMS M\&O 1998e. Cumulative Distribution Functions for the Temperature Threshold for the Onset of Carbon Steel Corrosion. B00000000-01717-0210-00015 REV 00. Las Vegas, Nevada. TBV-350.

CRWMS M\&O 1998f. Cumulative Distribution Functions for the Relative Humidity Thresholds for the Onset of Carbon Steel Corrosion. B00000000-01717-0210-00016 REV 00. Las Vegas, Nevada. TBV-350.

CRWMS M\&O 1998g. Parameters for Alloy C-22 Localized Corrosion Model. B00000000-017170210-00032 REV 00. Las Vegas, Nevada. TBV-349.

CRWMS M\&O 1998h. Supporting Electronic Media for "Creating Input Tables From WAPDEG for RIP, REV. 00." REV. 00. Self-extracting archive 210-13REV00.exe on 3.5" PC diskette. Las Vegas, Nevada. MOL.19980716.0559.

CRWMS M\&O 1998i. Software Routine Report for WAPDEG (Version 3.09). DI: $30048-2999$ REV 02. Las Vegas, Nevada: Mon, K.G.

CRWMS M\&O 1997. Final Report on Waste Package Degradation Expert Elicitation Project. Las 
Vegas, Nevada, August 15. MOL.19980218.0231. MOY-971120-07-02.

Golder Associates, 1998. RIP Integrated Probabilistic Simulator for Environmental Systems Theory Manual and User's Guide. March. Redmond, California: Golder Associates, Inc.

Marsh, G. P. and Taylor, K. J. 1988. "An Assessment of Carbon Steel Containers for Radioactive Waste Disposal." Corrosion Science, 28, 289-320. 223393.

Marsh, G. P.; Taylor, K. J.; and Sooi, Z. 1988. The Kinetics of Pitting Corrosion of Carbon Steel. SKB Technical Report 88-09. Stockholm, Sweden. February. NNA.19891026.0015.

Pasupathi, V. 1997. CRM Degradation Models-Update. CRWMS M\&O (Civilian Radioactive Waste Management System, Management and Operating Contractor) Interoffice Correspondence. LV.WP.VP.12/97-268, December 22. MOY-971231-11. TBV-349.

Pendleton, M.W. 1998. Waste Package Degradation Expert Elicitation Revised Preliminary Inputs Received by March 31, 1998. CRWMS M\&O Interoffice Correspondence, LV.EI.MWP.04/98-017, April 13. TBV-323.

\subsection{Attachments}

N/A. 